STUDIA ROMANICA POSNANIENSIA

UAM Vol. 42/1 Poznań 2015

TERESA MURYN

Université Pédagogique de Cracovie

teresa.muryn@gmail.com

\title{
L'ARTICLE COMME EXPOSANT DE L'ASPECT ? LA VALEUR ASPECTUELLE DU SN ET LA VALEUR ASPECTUELLE D'UNE COLLOCATION VERBO-NOMINALE
}

\begin{abstract}
Teresa Muryn, L'article comme exposant de l'aspect? La valeur aspectuelle du SN et la valeur aspectuelle d'une collocation verbo-nominale [An aspectual analysis of the noun phrase], Studia Romanica Posnaniensia, Adam Mickiewicz University Press, Poznań, vol. XLII/1: 2015, pp. 49-62. ISBN 978-83-232-2854-7. ISSN 0137-2475. eISSN 2084-4158. DOI: 10.14746/strop.2015.421.004
\end{abstract}

The definition of aspect as a semantic category enables aspectual analysis of the NP to be carried out. Noun phrases are viewed as aspectually equivalent to VPs. In both cases, the analyses are likely to be conducted along the same set of principles of aspectual decomposition. The point is to ascertain whether and how the category of aspect may contribute to the contrastive description of articles between a language with no articles and an article-language.

Keyw ords: semantics, syntax, verbal aspect, article, noun phrase, collocation.

L'analyse contrastive des langues slaves et des langues romanes est dominée surtout par deux domaines : celui de la détermination et de la quantification d'une part et de l'aspect de l'autre. Toutefois la définition de l'aspect comme catégorie sémantique ou trait inhérent du concept, permet de soumettre au calcul aspectotemporel non seulement le verbe, mais aussi le nom qui, à son tour, permet de situer l'article parmi les exposants de la catégorie d'aspect. Par conséquent, une réflexion portée sur l'aspect devrait s'appuyer sur une réflexion portée par la détermination ce qui pourrait faciliter, grâce à un critère supplémentaire, une analyse contrastive de l'article qui s'enrichirait ainsi de nouveaux arguments. Une telle analyse devrait distinguer entre le fonctionnement du SN dans une position d'argument et le fonctionnement du SN dans une collocation verbo-nominale. Si la valeur aspectuelle du $\mathrm{SN}$ dans ces positions avait un caractère de présupposition, le choix de l'article en tant qu'exposant de l'aspect pourrait aussi être traité dans ce cadre. L'analyse cidessous n'est qu'une hypothèse formulée (et possible) sur une base méthodologique que donne la grammaire à base sémantique, surtout dans la version de syntaxe sémantique proposée par l'école polonaise (K. Bogacki, S. Karolak, 1991). 


\section{LA DÉFINITUDE $V S$ L'INDÉFINITUDE. LA COMPLÉTUDE VS L'INCOMPLÉTUDE DU CONTENU CONCEPTUEL}

Les langues comme le français, disposant d'articles, exposants formels de définitude et d'indéfinitude, nommées langues à articles, se distinguent des langues comme le polonais, qui n'ont pas ces exposants et qu'on décrit comme langues sans articles. Dans les deux langues l'opposition ne concerne, à vrai dire, que les articles parce que d'autres déterminants qui existent en français comme adjectifs possessifs, démonstratifs, indéfinis, etc., ont leurs équivalents en polonais et leur fonctionnement dans les deux langues est similaire.

Un autre problème dans l'analyse contrastive entre les langues à articles et les langues sans articles est le fait que même dans les langues à articles le nombre d'articles et leur distribution n'est pas identique. Le français, par exemple, possède quatre articles : défini, indéfini, partitif et zéro. Confronté au système anglais à trois éléments, il démontre d'importantes différences dans leurs emplois. Le zéro d'article, par exemple, dont l'apparition dans les deux langues devrait être pareille, ne l'est pourtant pas et il n'existe aucune règle convaincante pouvant expliquer le phénomène (Karolak, 2002). Des langues sans articles, confrontées à des langues à articles ont donc encore un problème à surmonter : extraire dans leur système sans articles, les équivalents des structures à zéro d'articles (donc sans articles) de ces premières. Qui plus est, extraire des structures qui varieraient d'une langue à une autre !

Deux grandes théories, nées sur la base des réflexions logico-sémantiques concernant la nature de la langue, décrivent l'article en exploitant les notions d'intension et d'extension du nom.

La théorie extensionnelle, dite aussi référentielle, applique le critère de la supposition d'unicité et de la supposition pragmatique de la connaissance de l'objet désigné par les locuteurs. Le nom possède l'intension, c'est-à-dire le contenu conceptuel, tandis que sa référence ou l'extension actuelle lui est imposée dans une occurrence par des actualisateurs, entre autres, des articles. Pour cette théorie, l'opposition définitude vs indéfinitude est donc cruciale, aussi bien que les notions de présupposition d'existence et de présupposition de totalité (Kleiber, 1983) dans la description de l'article défini considéré comme primaire.

La théorie intensionnelle de l'article proposée par S. Karolak utilise la notion d'intensité du nom. Le nom représente son contenu conceptuel (intensité) en totalité, mais quand il est employé dans une phrase, il peut représenter seulement sa partie (Karolak, 2001 : 442). Des concepts uniques et généraux et les noms qui les représentent fonctionnent dans des contextes généraux ou spécifiques. La relation entre la valeur du nom et la valeur de la position syntaxique ouverte par le contexte s'exprime à l'aide de deux règles sémantiques, c'est-à-dire, la règle de la complétude intensionnelle (complétude du contenu conceptuel) et la règle de l'incomplétude intensionnelle (incomplétude du contenu conceptuel) (Karolak, $2001:$ 445). La 
théorie intensionnelle, pareillement à la théorie référencielle, postule le caractère primaire de la coïncidence de la valeur du nom et de la valeur de la position syntaxique qu'il occupe (complétude) pour des positions d'arguments, conformément à la définition de l'argument en tant qu'individu. Avec des règles particulières, elle explique la relation d'inclusion de deux valeurs, expliquant ainsi l'emploi de l'article indéfini ou le manque d'article. Dans cette théorie, l'opposition définitude/indéfinitude trouve une description sur le niveau universel, sémantique, égale aux langues à et sans articles.

Bien que les deux théories affirment que le contexte phrastique dans lequel le nom fonctionne doit être pris en compte, surtout la position syntaxique qu'il occupe, le plus souvent, elles trouvent la solution de l'article dans le SN même, dans sa complétude ou sa référence. En distinguant entre l'emploi argumentatif et prédicatif du SN, elles « égalisent » pourtant toutes les positions d'arguments impliquées par le prédicat en les ouvrant à la complétude. Il semble que l'application d'un critère d'ordre aspecto-pragmatique par rapport aux SN et le fonctionnement du SN dans la phrase nuance un peu cette vision de choses. La différence dans le traitement « classique » de positions d'arguments consisterait à distinguer l'emploi du nom dans une position indépendante et dans une collocation verbo-nominale. Par cette dernière, il faudrait comprendre non seulement les constructions $\mathrm{V}_{\mathrm{SUPP}}+$ Nom traditionnellement considérées comme ce type de collocation, mais aussi les séquences $\mathrm{V}_{\mathrm{f}}+$ Nom, c'est-à-dire, le verbe et son complément. Le nom dans la position syntaxique de complément construit un tout aspectuel avec le verbe qui l'implique et lui sert, au moins dans le contexte spécifique, de borne. Les deux d'ailleurs, le verbe et le SN, forment des configurations aspectuelles étant donné qu'un syntagme nominal a une valeur aspectuelle propre. D'autres positions syntaxiques (sujet, bénéficiaire) ne participent pas à la création de la configuration aspectuelle, elles servent à expliciter d'autres contenus impliqués, entrant ou non dans une relation imposée par le prédicat et c'est sur elles que semble peser la présupposition de complétude/définitude.

\section{L'ASPECT, UNE CATÉGORIE SÉMANTIQUE}

L'aspect a divisé les langues selon l'existence ou non, dans leurs systèmes, d'exposants formels de cette catégorie. Cette fois, les langues slaves, et par conséquent le polonais également, traitées comme langues aspectuelles se distinguent des langues sans exposants aspectuels, comme, par exemple, le français. Pour ce dernier, la catégorie de l'aspect, formellement liée à la catégorie du temps, fonctionne dans le système d'oppositions accompli vs non accompli (Benveniste, Guillaumme) qu'on a souvent faussement identifié au système polonais perfectiflimperfectif (dokonaność/niedokonaność) en générant des confusions et des erreurs d'interprétation. 
Dans ce cas, comme dans le précédent, poser le problème au niveau sémantique s'est avéré une solution satisfaisante et cela a permis d'établir des équivalences au niveau universel des concepts et plutôt qu'au niveau idiomatique d'exposants formels.

La théorie sémantique de l'aspect trouve ses origines dans les travaux de G. Guillaume qui distingue le temps impliqué du temps expliqué du verbe. Enrichie de la méthode combinatoire proposée par F. Antinucci et L. Gebert, elle a trouvé sa description complète dans les travaux de S. Karolak. L'originalité de cette théorie repose dans le fait qu'elle rejette la distinction traditionnelle entre aspect lexical (modalité d'action propre aux sémantèmes) et aspect grammatical (propre aux grammèmes) et n'accepte pas d'identifier les formes simples avec des aspects simples. En effet, l'aspect est un trait inhérent du concept. Il existe deux concepts aspectuels dits primitifs : celui de continuité (imperfectif simple) et celui de non-continuité (perfectif simple). Ils permettent de faire dériver des configurations aspectuelles (aspects complexes) avec une dominante continue ou non-continue. Les formes constituées par des sémantèmes simples peuvent donc être aspectuellement simples ou complexes. Il faut « traiter l'aspect comme constituant de la phrase, c'est-à-dire dépasser le cadre de la structure interne. Un verbe peut se révéler insuffisant comme marque de tel ou tel aspect, exigeant alors l'adjonction d'un autre constituant de phrase ». Le constituant extra-verbal peut participer à la constitution de l'aspect ou bien désambigüiser celui-ci quand différentes lectures d'une forme verbale sont possibles (Karolak, $2008: 127-130$ ).

\section{LA VALEUR ASPECTUELLE DES NOMS}

L'aspect ainsi défini - comme catégorie sémantique - a permis d'appliquer une analyse aspecto-temporelle non seulement à des verbes mais aussi à d'autres classes morphologiques, comme, par exemple, les noms, les adjectifs, les prépositions, etc. Comme l'analyse aspecto-temporelle se rapporte à la proposition entière, elle exige qu'on prenne en compte toutes ses réalisations possibles; non seulement la phrase mais aussi le syntagme nominal qui est son équivalent formel. Dans cette deuxième situation, quand le SN est soumis à l'analyse aspectuelle, il faut chercher d'autres exposants de temps et d'aspect que dans le cas du verbe. En même temps, prenant en considération les fonctions syntaxiques que peut assumer un nom, il faut supposer que sa valeur aspectuelle est calculée à partir de sa relation avec d'autres constituants de la phrase dans laquelle il fonctionne.

Dans le cas donc, où l'analyse aspectuelle s'applique au syntagme nominal, l'opposition exprimée par des articles défini-indéfini-partitif-zéro acquiert une interprétation supplémentaire. Dans cette opposition, pertinente pour le contexte spécifique qui implique une limitation (contrairement au contexte générique), le choix de l'article peut s'expliquer par l'explicitation de cette limite. 
En acceptant, par analogie au verbe, que le sémantème du nom soit porteur de l'aspect et que les exposants aspectuels puissent se trouver en dehors du nom, il faut admettre aussi que le calcul aspectuel du nom se fera de façon semblable à celui du verbe. D'abord, la valeur aspectuelle du nom sera établie sur la base de son sémantème avant d'être calculée à partir de son fonctionnement dans le syntagme nominal avec d'autres expansions (s'il y en a). De plus, puisque le syntagme nominal dans une phrase coopère avec d'autres éléments qui la constituent, la question sera de savoir, analogiquement au verbe, si le SN examiné forme avec un autre constituant une collocation à l'aspect complexe (une configuration aspectuelle) ou bien, s'il fonctionne comme constituant aspectuellement indépendant dont la fonction est de saturer une position d'argument.

\section{LES NOMS : LEUR CLASSEMENT ET LA VALEUR ASPECTUELLE}

Les classements traditionnels et communément acceptés des noms communs les divisent en deux groupes ${ }^{1}$, très imprécis, de noms concrets et de noms abstraits. Dans le premier (noms concrets), selon le critère sémantico-référentiel, on a rangé les noms désignant des objets physiques, des êtres et des choses. Dans le second (noms abstraits), on a réuni les noms décrits selon un critère sémantico-morphologique, les noms de propriété, processus, etc., dans la plupart des cas dérivés d'adjectifs et de verbes. Il n'y a pas de place dans ce classement pour les noms dits abstraits qui fonctionnent pourtant avec un sens concret et se comportent comme tels (p. ex. une beauté, une bonté, une bêtise). Dans cet article, nous allons adopter la définition des noms abstraits et des noms concrets qui prend en compte ce double emploi du nom abstrait en précisant pourtant que la différence est perceptible au niveau du syntagme et du référent et non pas au niveau du sémantème. Il s'agit d'une définition syntaxique qui permet de distinguer les noms qui représentent la structure sémantique sous-jacente dans sa totalité (les noms abstraits) des noms qui ne représentent qu'une des positions d'argument de la structure sous-jacente, celle que le prédicat a résorbée (les noms concrets).

Les verbes (ou les adjectifs) donnent donc lieu a deux types de dérivation (Muryn, 1999). La première produit des noms qui permettent l'explicitation de tous les arguments impliqués par le prédicat tandis que la seconde bloque une des positions d'argument. La première ne change ni la valence du prédicat ni son contenu sémantique, la seule différence entre les deux formalisations (verbe vs nom) est d'ordre syntaxique. La nominalisation n'est ici qu'une opération purement formelle : à titre d'exemple, comparons la phrase (1) au SN (1'). L'exemple (1) nous donne la chaîne argumentative du prédicat formalisé par le verbe promettre :

\footnotetext{
${ }^{1}$ Nous n'évoquons que le classement qui est intéressant du point de vue de l'analyse aspectuelle.
} 
(1) Pierre a promis de venir demain. / Piotr obiecat przyjść jutro / że przyjdzie jutro.

Cette phrase, avec le prédicat nominalisé, peut prendre la forme du SN équivalent qu'illustre l'exemple (1') :

(1') la promesse de Pierre de venir demain / ze przyjdzie jutro (obietnica Piotra przyjścia jutro...).

La seule modification introduite par la transformation nominale concerne le comportement syntaxique du SN : il ne peut fonctionner que dans une position ouverte par le prédicat d'ordre supérieur. Ces noms peuvent être considérés comme abstraits.

La classe des noms concrets, en revanche, traditionnellement compris comme des noms d'objets perçus par les sens enfant/dziecko, voiture/samochód, chien/pies, pays/kraj, montagne/góra doit s'enrichir des noms dérivés à partir de verbes et d'adjectifs qui désignent un de leurs arguments. Issus de l'opération dite de résorption de la position d'argument, ces noms se caractérisent par le fait qu'ils ont une chaîne argumentative réduite d'un argument par rapport aux prédicats dont les sémantèmes ils héritent : celui dont la position a été résorbée, p. ex. déclaration/ deklaracja, promesse/obietnica, souci/zmartwienie, hypothèse/hipoteza, condition/warunek, beauté/piękność, gentillesse/uprzejmość, bêtise/głupstwo. Ces noms désignent des objets de pensée (ou, selon la proposition de G. Gross, des objets logiques) comme p. ex. le nom promesse dans son emploi concret dans le SN (1'’) :

\section{(1',) une promesse de Pierre / obietnica Piotra.}

Le SN décrit ici le contenu de la position d'argument : ce que Pierre a promis / to, co Piotr obiecat (contrairement au nom abstrait, sans résorption, qui réfère au fait : le fait que Pierre a promis de venir / fakt, że Piotr obiecat przyjśś). Les SN de ce type présupposent l'existence du contenu de la position résorbée, ils peuvent donc se comporter comme tous les SN concrets, fonctionner dans des positions d'arguments, mais aussi former des collocations verbo-nominales auxquelles ils apportent une notion supplémentaire : celle d'antériorité de la structure dont ils sont dérivés.

Un autre critère regroupe des noms dans les classes de noms comptables et de noms massifs. Les noms comptables renvoient à des objets ayant des dimensions spatiales mesurables, p. ex. livre/książka, arbre/drzewo, à des objets mentaux (noms avec résorption) idée/pomyst, pensée/myśl, souvenir/wspomnienie, imprudence/nieostrożność, mais aussi à des états de choses mesurables dans le temps comme les événements, les actions, p. ex. cri/krzyk, explosion/wybuch, etc. Les noms massifs dénotent des substances sans forme, p. ex. eau/woda, farine/maka, air/powietrze, mais aussi, étendus dans le temps sans limites, des états, des propriétés, des attitudes, etc., p. ex. mądrość/sagesse, gaité/wesołość, couragelodwaga... 
Quand on applique aux noms évoqués le critère aspectuel exploitant les primitifs aspectuels de continuité et de non-continuité, on se rend compte que leur répartition n'est pas la même qu'avec les critères traditionnels. Tous les noms, indépendamment de leur caractère abstrait ou concret, représentent des concepts continus ou discontinus, simples ou complexes (configuration d'aspects). Les noms concrets comptables et massifs sont déjà répartis en deux groupes aspectuels des noms noncontinus et des noms continus. Les sémantèmes des noms dits abstraits véhiculent toujours la même valeur aspectuelle que les sémantèmes de verbes ou d'adjectifs dont ils ont été dérivés : le sémantème du nom amour/miłość est continu et simple comme l'est celui du verbe aimer/kochać, le sémantème du nom chute/upadek en revanche est non-continu et simple comme celui du verbe tomber/upaść. Le problème est de savoir comment classifier les noms avec résorption. La réponse paraît évidente : il s'agit d'une configuration d'aspects. Prenons comme exemple l'adjectif vrai dont le sémantème représente un concept continu simple. Employé dans le syntagme abstrait, représentant la totalité de la structure sous jacente, il véhicule sa valeur aspectuelle basique sans aucune modification :

(2) La vérité de cet aveu est incontestable / Prawda tego wyznania jest niepodważalna.

Pourtant, quand il est employé dans la version avec résorption, le nom réfère au contenu de l'aveu qui en fait un nom concret :

(2') Ce qu'il a avoué est une vérité incontestable / To, co wyznat, jest niepodważalna prawda.

Dans cet emploi, le sémantème a conservé la valeur continue et simple d'origine, mais le concept s'est trouvé sous la domination d'un autre - celui du contenu de l'aveu - qui a limité de l'intérieur sa portée. Les deux aspects participent à une configuration limitative qui, pour être reconnue, doit avoir des données contextuelles. C'est ainsi que l'opposition entre les noms abstraits et les noms concrets d'objets mentaux n'est pertinente qu'au niveau des syntagmes et l'article indéfini introduisant le nom avec résorption devient un exposant de la configuration aspectuelle à dominante non-continue dans les langues à articles. Une borne intérieure limitant ces prédicats est d'ailleurs propre aux positions d'arguments qu'on pourrait voir comme supports obligatoires de la prédication, comme par exemple celle du porteur d'une qualité, du patient, de la cause pour des prédicats résultatifs, etc. (p. ex. une beauté - ce qui est beau, une pensée - ce qu'on a pensé, une gentillesse un acte gentil, un chagrin - cause du chagrin). De toute façon, ce qui est crucial pour cette configuration aspectuelle, c'est le fait que la dominante non-continue doit être fournie par le contexte : le syntagme doit sa lecture aspectuelle grâce à la coopération avec le verbe, avec lequel il forme une collocation. L'article indéfini n'est qu'un exposant supplémentaire de la configuration : la valeur du syntagme se 
reconnait donc, en polonais et en français par le calcul des données contextuelles comme cela a lieu dans les exemples (3) et (3') :

(3) Il n'a eu qu'un plaisir pendant ce voyage : goûter à la liberté. / Miat w tej podróży tylko jedna przyjemność: zasmakować wolności.

vs

(3') Il appréciait le plaisir de goûter à la liberté que ce voyage lui donnait. I Cenit przyjemność smakowania wolności, która ta podróż mu dawała.

En résumant les remarques précédentes et en appliquant le critère aspectuel, nous pouvons constater que les sémantèmes des noms se divisent en deux groupes de concepts continus (simples et complexes) et de concepts non-continus (simples et complexes), indépendamment du critère qui les divise en noms abstraits et en noms concrets :

1. Les sémantèmes continus sont des substances et des états de choses continus (propriétés, activités, attitudes, actions téliques, des états résultatifs, etc.).

2. Les sémantèmes non-continus sont des objets mesurables, des objets mentaux, des états de choses non-continus (événements, actions, etc.).

Tous ces noms, en fonctionnant dans différents types de contextes, peuvent conserver la valeur aspectuelle de leurs sémantèmes ou bien former différents types de configurations aspectuelles : pour chaque configuration pourtant il y aura des traces dans le contexte, c'est-à-dire, chaque aspect participant à la configuration aura son exposant formel. Le contexte non seulement explicite la valeur avec laquelle le nom est employé, mais semble aussi imposer une valeur aspectuelle de départ, on pourrait dire qu'il la présuppose.

\section{L’EMPLOI GÉNÉRIQUE ET L’EMPLOI SPÉCIFIQUE DU NOM}

La situation où la valeur aspectuelle du nom employé est égale à la valeur de son sémantème est propre au contexte générique. Dans cet emploi pourtant l'opposition des articles se neutralise, elle est pertinente pour le contexte spécifique.

Le contexte spécifique est soumis aux contraintes temporelles et spatiales. L'apparition d'un concept dans un tel contexte implique déjà la quantification : l'existence d'un référent dans le temps réel implique la limitation de cette existence. Pour les noms, cela signifie que, employés dans les SN spécifiques, ils véhiculent une valeur aspectuelle non-continue. Par conséquent, il semble autorisé de dire que les articles, indéfini et partitif, servent d'exposants aspectuels et expriment une valeur non-continue du nom employé dans le syntagme qu'ils précèdent. Nous réservons cette analyse aux noms en positions « après le verbe » : en position d'objet direct 
après le verbe plein et en position de nom prédicatif après le verbe support dans les collocations verbo-nominales. Du point de vue aspectuel, ces positions semblent symétriques et c'est là que l'article partitif et l'article indéfini peuvent s'opposer.

\section{L'ARTICLE PARTITIF ET L'ARTICLE ZÉRO COMME EXPOSANTS DE LA VALEUR ASPECTUELLE DU SYNTAGME}

En acceptant que, dans un contexte spécifique, le SN renvoie à un fragment de la réalité, nous devons décrire l'emploi de l'article indéfini devant les noms à valeur non-continue en termes de neutralisation : cet article reflète seulement la valeur aspectuelle du sémantème. Cependant les noms à valeur continue employés dans ce contexte doivent apparaître dans une configuration à dominante limitative. Dans les langues à articles, ces derniers doivent rendre compte du changement opéré dans le $\mathrm{SN}$ : l'article indéfini et l'article partitif paraissent donc parfaitement aspectuels - ils véhiculent l'information que le référent auquel renvoie le nom a des limites spatiales ou temporelles. La limite ou, plutôt, la borne s'interprète selon le type de concept, sa valeur aspectuelle et sa coopération avec d'autres concepts. À titre d'exemple (et parmi d'autres réalisations possibles), le verbe marcher représente un concept aspectuellement simple (une activité) ; le SN une marche renvoie à un intervalle dans lequel marcher se réalise dans un temps réel. On peut l'envisager aussi comme une durée limitée $=$ deux heures de marche ou résultative : une marche de deux heures $^{2}$. Dans ces exemples la borne est temporelle, imposée de l'extérieur, conforme à la valeur aspectuelle du concept auquel elle s'impose.

Les noms dits d'objets mentaux ont une borne sémantique, autrement dit, ils sont limités sémantiquement, de l'intérieur. La limite de la durée est alors imposée par le caractère discret de leur argument : l'acte de déclaration, par exemple, est borné par la longueur de ce qu'on a déclaré. Une déclaration, le $\mathrm{SN}$ à valeur noncontinue, est donc créée par l'opération de résorption de la position d'argument à fonction aspectuelle de borne intérieure. Par conséquent, le nom devient une unité discrète. Il est dérivé à partir d'une structure à dominante non-continue, résultative, qui décrit l'action qui a atteint son terme. Ce SN renvoie non seulement à un objet mental prévu par la position résorbée, mais aussi « à la mémoire » du caractère perfectif de sa structure d'origine. Celle-ci est perçue comme antérieure par rapport au prédicat supérieur régissant le SN en question. Pour conclure, ces SN à valeur non-continue, comme p. ex. une connaissance, une déclaration, un conseil, etc., sont porteurs de notion de non-continuité et d'antériorité. On peut le prouver en paraphrasant le SN en question employé en position d'argument impliquée par le prédicat supérieur comme dans l'exemple suivant :

\footnotetext{
${ }^{2}$ On voit bien la différence entre les deux SN quand on emploie un nom concret : quand on achète deux mètres de câble cela n'équivaut pas à l'achat d'un câble de deux mètres.
} 
(4) Votre déclaration a soulevé une discussion animée. = ce que vous aviez déclaré, a soulevé une discussion animée.

Les prédicats continus limités de l'extérieur représentent une configuration aspectuelle à dominante non-continue qui a deux exposants dans le $\mathrm{SN}$; le sémantème véhicule la valeur continue de base et l'article partitif ou indéfini celle de la dominante non-continue. Il informe que le nom est employé dans une valeur qui n'est pas conforme à sa valeur sémantique et qu'il faut chercher d'autres éléments dans le contexte qui justifient ce manque de coïncidence. Le SN précédé d'article partitif n'a pas d'autonomie, il dépend d'un autre élément qui impose la lecture spécifique et une borne à la continuité. La configuration limitative ne concerne pas le SN tout seul mais la collocation qu'il crée avec le verbe. C'est pour cette raison que le polonais n'a pas besoin d'article pour marquer le caractère complexe et non continu du SN, celui-ci se déduit (ou se calcule) de la valeur du sémantème du nom et celle du membre dominant dans la collocation comme c'est le cas des phrases (5-8) qui illustrent la configuration limitative, explicitée par l'article indéfini ou l'article partitif en français et déchiffrée grâce aux données contextuelles en polonais :

(5) Paul a toujours faim? Il a mangé un sandwich. / Pawet jest ciagle głodny? Jadt/zjadl kanapke.

(6) Paul a mangé de la viande bien qu'il préfère la salade. / Pawet jadt/zjadt mięso, chociaż woli sałatę.

(7) Il aura de la chance s'il s'en tire. / Będzie mial szczęście, jak z tego wyjdzie.

(8) Il a quand même manifesté de la générosité. / Mimo wszystko okazal wspanialomyślność.

Dans tous ces exemples le nom coopère avec le verbe. Dans le cas des séquences dites libres, du verbe avec son objet, le verbe à part une caractéristique aspecto-temporelle possède aussi un « riche » sémantème que le nom complète obligatoirement dans un contexte spécifique ; il lui sert de support concret, impliqué d'ailleurs sémantiquement. On doit manger quelque chose et cette implication est tellement évidente que sa réalisation formelle est souvent omise. Pourtant une inférence sémantico-aspectuelle permet de prévoir qu'on mange si longtemps que l'objet que l'on mange le permet ${ }^{3}$. Les substances, qui n'ont pas de forme, sont arbitrairement limitées à l'aide de portions qui leur donnent une existence réelle.

La même situation a lieu dans le cas de noms continus étendus dans le temps comme les états, propriétés ou attitudes. Leurs emplois spécifiques se rapportent à cette parcelle du temps où une propriété, état, attitude, etc., se manifestent. La borne peut être temporelle et explicite, mais elle peut être donnée aussi par

\footnotetext{
${ }^{3}$ On peut bien sûr arrêter arbitrairement de manger, voir l'action comme télique, p. ex. Il mange un sandwich/ de la viande/ a de la chance / manifeste de la générosité, etc., mais ce sont des configurations plus complexes dérivées de la configuration limitative.
} 
l'intermédiaire d'une action ou d'un acte par lequel le trait s'extériorise. Ces SN entrent dans un autre type de collocation : les noms traités comme prédicatifs forment une collocation verbo-nominale avec des verbes supports. Le nom se trouve sous la dominance d'un autre prédicat, sémantiquement pauvre, réduit parfois au concept aspectuel (action, acte, état). Formellement, le SN est introduit par un verbe support qui apporte à la construction complexe sa valeur aspectuo-temporelle. Le choix du verbe support est quand même assez libre, parfois jusqu'à la confusion avec le verbe plein (p. ex. bombarder, multiplier, manifester, etc.). La valeur aspectuelle d'une construction de ce type est calculée à partir de deux unités autonomes qui la composent : le verbe support et le $\mathrm{SN}$. La construction $V_{\text {sup }}+S N$, du point de vue syntaxique, ressemble à la construction $V+S N$ que forme un verbe plein avec son objet direct, de toute façon, l'une est symétrique à l'autre, la seule différence se trouve dans le fait que les deux structures sémantiques, lexicalement, répartissent leur sens autrement. Si le verbe plein n'a besoin de son argument direct que pour compléter son sens, dans la collocation avec le verbe support, la situation est inverse. Dans les deux cas pourtant, le verbe construit un tout aspectuel avec le SN et, dans les deux cas, la valeur limitée du SN est imposée par l'emploi spécifique du verbe.

Il reste le problème de constructions $V_{\text {sup }}+0+N_{\text {préd }}$ où le verbe support introduit le nom sans article (ou avec article zéro, comme le propose J.-C. Anscombre, 1986, 1990, 1991) du type prendre connaissance de, donner ordre de, faire allusion à, livrer bataille, faire tour, faire hommage, faire escorte. Le mécanisme qui permet de faire dériver ces constructions et qui opère tant sur le niveau formel que sémantique assigne à un concept les traits aspectuels dont il ne disposait pas avant. La construction qui en est le résultat est formellement complexe : le nom ne véhicule que la valeur aspectuelle de son sémantème, le verbe support est son grammème, " sa désinence ». Dans cette dérivation, on crée une sorte de verbe complexe, polylexical. Le nombre de verbes supports qui apparaissent dans ce type de construction est assez réduit : le plus souvent faire, prendre, avoir et donner. Ces verbes semblent renvoyer aux notions de prototypes verbaux tels qu'événement, action ou état. Dans ce cas, le nom est vraiment actualisé par le verbe support qui le fait conjuguer, comme le dit Gaston Gross. Nous proposons d'attribuer à ces constructions le statut de constructions figées, issues du procédé de dérivation lexicale.

Ces constructions sont à distinguer des autres où le nom fonctionne aussi sans article, mais elles fonctionnent dans des structures complexes qui ont subi plusieurs transformations. Elles méritent, d'après nous, une attention particulière parce que beaucoup d'entre elles témoignent d'une transformation nominale équivalente à la transformation infinitive éliminant la redondance dans des phrases complexes coréférentielles. Il s'agit des syntagmes prépositionnels (et de leurs équivalents polonais qui se comportent de la même façon) de types :

(9) De joie, il a sauté (G. Gross). I Z radości podskoczyt.

(10) De peur, il a fuit. / Ze strachu uciekt. 
(11) Je ne dirai pas non par gentillesse. / Nie odmówię przez uprzejmość.

(12) Nous accomplissons cette tâche avec patience, mais aussi avec plaisir. / Wykonujemy to zadanie z cierpliwościa, ale także z przyjemnościa.

(13) De colère, il a rougi. / Ze ztości poczerwieniat.

Ce ne sont que des ellipses syntaxiques qui n'apparaissent que dans des phrases complexes qui représentent des structures sémantiques complexes coréférentielles impliquées par des prédicats d'ordre supérieur. Le SP auquel on attribue la fonction syntaxique de complément circonstanciel, y représente la proposition subordonnée. Quand les deux propositions, dominée (subordonnée) et dominante (principale), sont coréférentielles, la dominée ne conserve au niveau formel, au niveau de l'expression, que ces éléments qui sont nécessaires sémantiquement et en élimine d'autres dont la répétition pourrait nuire à l'économie de la communication. Il faut souligner ici le rôle de la préposition : la lecture aspectuelle de la séquence entière (du SP) se déduit de la valeur du sémantème nominal et de celui de la préposition en coopération avec le verbe de la proposition principale (Muryn, 2010). Avec la participation du contexte, il est facile de reconstruire tous les éléments d'une telle construction :

(10') Puisqu'il avait peur, il a fui. / Ponieważ się bat, uciekt.

(10") Pourquoi vous avez fui? - De peur. / Parce que j'ai eu peur. / Dlaczego Pan uciekt? - Ze strachu. Ponieważ się balem.

(12') Nous accomplissons cette tâche avec patience. Nous sommes vraiment patients en accomplissant cette tâche. / Wykonujemy to zadanie z cierpliwością. Jesteśmy naprawdę cierpliwi, wykonując to zadanie.

Pour ces structures qui n'existent pas en dehors du cadre phrastique qui les englobe et qui n'ont aucune autonomie, l'omission de l'article n'est due qu'à une opération formelle d'effacement, aucune modification au niveau sémantique n'a eu lieu.

\section{SANS CONCLUSION}

Dans l'analyse précédente, nous avons pris en compte la position du nom dans les collocations qu'il produit avec le verbe. Notre but était de démontrer que dans les deux cas analysés le SN forme un tout aspectuel avec le verbe qui l'introduit. Les deux, le verbe et le nom, participent à la construction de la configuration aspectuelle limitative et de ce point de vue, ils sont inséparables. La position du nom dans cette collocation serait donc différente de celle de sujet par exemple (ou de bénéficiaire, etc.). Fonctionnant comme exposant de borne aspectuelle sémantiquement impliquée, il pourrait être décrit en tant qu'argument interne de la structure prédicative, tandis que le sujet (ou autres) aurait le statut de vraie position d'argument. Pour l'argument interne dans le contexte spécifique, on supposerait donc un article indéfini/partitif comme un exposant primaire de l'aspect, tandis que d'autres posi- 
tions d'argument supposeraient l'article défini conformément au besoin de complétude/définitude imposé pour une position référencielle. Avec une opposition semblable de présuppositions, on pourrait s'attendre, dans les langues sans articles, à l'apparition d'un déterminant ou à une modification syntaxique quand, pour des raisons différentes, elle n'opère pas, par exemple :

(13) Dziewczyna kupiła książkę. / La jeune fille a acheté un livre.

(13') Jakaś dziewczyna kupiła ksiązę. / Une jeune fille a acheté un livre.

(13') Książke kupiła właśnie jakaś dziewczyna. I Le livre vient d'être acheté par une jeune fille.

Il ne s'agit ici que d'une hypothèse de travail, d'une solution possible au problème de l'article dans une analyse contrastive. Elle doit prendre en compte non seulement le calcul sémantico-syntaxico-aspectuel mais aussi d'autres données comme par exemple la structure thème-rhème de la phrase. Il semble donc qu'il serait plus juste de voir cette analyse sur le plan aspecto-pragmatique.

L'analyse aspectuelle de syntagmes nominaux, elle-même, apporte beaucoup de problèmes particuliers, comme celui, entre autres, d'exposants de configurations aspectuelles dans le SN ou d'une analyse aspectuelle des SN représentant des structures sémantiques complexes. La recherche d'une unité aspectuellement homogène, le phénomène de l'inférence pris en compte, nous mène à la recherche de séquences qui, parfois, sont plus grandes que les positions syntaxiques traditionnelles.

\section{RÉFÉRENCES}

ANSCOMBRE, Jean-Claude (1986) : « L'article zéro en français : un imparfait du substantif ? ». Langue française 72 : 4-39.

ANSCOMBRE, Jean-Claude (1990) : " Article zéro et structuration d'événements ». Dans : Michel Charolles, Sophie Fisher, Jacques JAYEZ (éds.), Le discours : représentations et interprétations, Nancy : P.U.N., 265-300.

Anscombre, Jean-Claude (1991) : « La détermination zéro : quelques propriétés ». Langages $102: 103-124$.

AnTINUCCI, Francesco \& Gebert, Lucyna (1977) : « Semantyka aspektu czasownikowego ». Studia Gramatyczne $1: 7-43$.

BOGACKI, Krzysztof (2005) : « Le traitement de l'article dans la traduction automatique du polonais vers le français ». Neophilologica $17: 43-57$.

BogaCKI, Krzysztof \& KAROLAK, Stanisław (1991) : "Fondements d'une grammaire à base sémantique ». Lingua e Stile XXVI : 3.

KAROLAK, Stanisław (1994) : « Le concept d'aspect et la structure notionnelle du verbe ». Studia Kognitywne 1, Warszawa : Slawistyczny Ośrodek Wydawniczy.

KAROLAK, Stanisław (2001) : «Jak tłumaczyć coś, co nie istnieje? ». Dans : Od semantyki do gramatyki, Warszawa : Instytut Slawistyki PAN, 439-459. 
KAROLAK, Stanisław (2004): Rodzajnik francuski w ujęciu funkcjonalnym, t. 1. Kraków: Collegium Columbinum.

KAROLAK, Stanisław (2008) : «Remarques sur l'équivalence du passé imperfectif polonais et des temps passés en français », VERBUM XXX, 2-3 : 125-147.

KLEIBER, Georges (1983) : «Article défini, théorie de la localisation et présupposition existentielle ». Langue française $57: 87-105$.

MEJRI, Salah (2008) : "Construction à verbes supports, collocations et locutions verbales». Dans : Pedro Mogorron HuerTa \& Salah MeJRI (éds.), Las construcciones verbo-nominales libres y fijas. Aproximación contrastiva y traductológica, 191-202.

MURYN, Teresa (1999): Le syntagme nominal abstrait et la cohérence discursive. Kraków: Wydawnictwo Naukowe WSP.

Muryn, Teresa (2006) : «L'expression de l'aspect limitatif dans le SN ». Synergies Pologne 2: $100-114$.

Muryn, Teresa (2009) : «Comment calculer la valeur aspectuelle d'un SN ? ». Synergies Pologne $6: 113-127$.

MURYN, Teresa (2010) : «La préposition comme exposant de l'aspect dans les constructions prép $+\mathrm{N}$ à valeur causale. Une analyse contrastive français-polonais ». Dans : Iva NovAKovA \& Elena Dontchenko (éds.), Grammaire et lexique : regards croisés, Astrakhan-Grenoble : Maison d'édition de l'Université d'Astrakhan, ELLUG, Université Stendhal, Grenoble, 93-115.

Muryn, Teresa (2011) : « Le nom, le Syntagme Nominal, la construction Vsup+N et leur valeur aspectuelle. Une analyse contrastive français-polonais ». Romanica Cracoviensia 11 :292-299.

MurYN, Teresa (2011) : «La détermination en polonais ; un déterminant zéro ? ». Neophilologica $22: 96-107$.

Muryn, Teresa (2013) : "Le rôle de la détermination du nom dans le calcul aspectuel sur l'exemple de quelques verbes polonais préfixés par $n a-$. Une analyse contrastive polonaisfrançais ». Neophilologica $25: 128-140$.

VetUlani, Grażyna (2010): Rzeczowniki predykatywne języka polskiego. W kierunku syntaktycznego stownika rzeczowników predykatywnych. Poznań : Wydawnictwo Naukowe UAM.

VetUlANI, Grażyna (2012) : Kolokacje verbo-nominalne jako samodzielne jednostki języka. Syntaktyczny słownik kolokacji werbo-nominalnych języka polskiego na potrzeby zastosowań informatycznych. Poznań : Wydawnictwo Naukowe UAM. 